\title{
Grouping by Visual Appearance of Construction and Demolition Waste for Sorting Time Reduction with the Aim of Removing Asbestos-Containing Materials
}

Hiroshi Asakura* and Kei Nakagawa

Graduate School of Fisheries Science and Environmental Studies, Nagasaki University, Nagasaki, Japan

\begin{abstract}
As asbestos-containing waste from building demolitions has to undergo special treatment prior to recycling or final disposal, the demolition of buildings and the sorting of waste materials are conducted after checking for asbestos content in the construction materials (according to ISO, EPA or JIS). However, as solid waste is usually a mixture, it is possible that asbestos-containing material (ACM) is present in construction and demolition waste (CDW) that is transported to an intermediate treatment facility for CDW. In addition, the presence of ACM in disaster waste cannot be avoided. Therefore, a rapid method for the determination of asbestos at an intermediate treatment facility for CDW is required. In this study, the separation efficiency and the sorting time of CDW particles by grouping by visual appearance (GVA) were determined. In the case that the separation efficiency by GVA in this study was equivalent to that by visual observation with a loupe (DVL) in a previous study, the reduction of sorting time by GVA was evaluated. Newton's separation efficiency by GVA and recovery rate were equivalent to that by DVL for $5.1 \mathrm{~cm}^{2}$ observation. In this case, the sorting time by GVA was $1 / 7$ of that by DVL. Therefore, sorting time could be shortened by GVA under the condition of equivalent separation efficiency. In order to reduce the sorting time per worker to less than $1 \mathrm{~h} / \mathrm{t}$, only CDWPs having particle size larger than $12 \mathrm{~cm}$ for GVA or $20 \mathrm{~cm}$ for DVL for $5.1 \mathrm{~cm}^{2}$ observation should be subjected to sorting. Aiming to avoid diffusion of asbestos-containing waste, the authors suggest that grouping by visual appearance as a primary sorting step is effective to reduce sorting time of CDW from disaster waste or unknown origin.
\end{abstract}

Keywords: Asbestos; Construction demolition waste; Visual appearance; Visual observation; Sorting

\section{Introduction}

Material containing $>0.1 \mathrm{w} \%[1,2]$ or $>1 \mathrm{w} \%$ [3] asbestos is regarded as asbestos-containing material (ACM), and solid waste containing $>0.1 \mathrm{w} \%$ or $>1 \mathrm{w} \%$ asbestos is regarded as asbestos-containing waste. As asbestos-containing waste from building demolitions has to undergo special treatment prior to recycling or final disposal, the demolition of buildings and the sorting of waste materials are conducted after checking for asbestos content in the construction materials (according to ISO, EPA or JIS). However, as solid waste is usually a mixture, it is possible that ACM is present in construction and demolition waste (CDW) that is transported to an intermediate treatment facility for CDW. In addition, the presence of ACM in disaster waste cannot be avoided. Therefore, a rapid method for the determination of asbestos at an intermediate treatment facility for CDW is required.

As a long time is required to identify asbestos fiber by conventional laboratory methods, such as those adopted by ISO, those methods are unsuitable for intermediate treatment facilities for CDW. On the other hand, Saitama Prefecture has developed a rapid method for asbestos fiber determination, which involves visual observation [4]. However, the determination accuracy and time are unknown.

The main topics of previous studies on waste materials and asbestos included asbestos content in waste sludge [5], particle emission from solid waste [6], and detoxification of asbestos in solid waste [7-12]. As far as we know, there are few or no studies on the sorting of ACMs and non-asbestos-containing materials (non-ACMs). To realize sorting, it is necessary to understand the physical and chemical properties of waste (e.g., density or electromagnetic property) and to determine whether the waste contains asbestos. The purpose of studies on the identification of asbestos [13-16] is to develop an accurate method for asbestos analysis. Bassani et al. [17] reported the use of remote sensing to detect asbestos in roofing sheets, which enabled scanning of ACMs by batch in large urban areas. Some studies have employed an advanced analyzer with high accuracy [17]. However, in actuality, the preliminary analysis of asbestos content in construction materials from demolition work is not being conducted in many cases in Japan. Currently, one of the major sorting methods at intermediate treatment facilities for $\mathrm{CDW}$ is manual sorting with visual observation. Therefore, estimation of the separation efficiency by visual observation should be conducted first, followed by consideration of the adoption of analytical instruments with high accuracy.

The authors previously investigated the separation efficiency of ACM from CDW particles by adopting the method of determination by visual observation with a loupe (DVL) [18]. A long time was required to examine the entire surface of a CDW particle. However, because most of the asbestos fibers were uniformly exposed on the cross section, the authors found that high separation efficiency could be achieved by DVL of only half of the particle cross section, i.e., observation time could be shortened [19]. However, even though DVL was carried out on half of the cross section, there was no change in the time required for visual observation and sorting because all of the particles had to be observed with a loupe.

CDW particles sorted by DVL were characterized by visual

*Corresponding author: Asakura $\mathrm{H}$, Graduate School of Fisheries Science and Environmental Studies, Nagasaki University, Nagasaki, Japan, Tel: +81-95-8192760; E-mail: asakura_hiroshi@yahoo.co.jp

Received September 07, 2017; Accepted September 14, 2017; Published September 21, 2017

Citation: Asakura H, Nakagawa K (2017) Grouping by Visual Appearance of Construction and Demolition Waste for Sorting Time Reduction with the Aim of Removing Asbestos-Containing Materials. Int J Waste Resour 7: 298. doi: 10.4172/2252-5211.1000298

Copyright: @ 2017 Asakura H, et al. This is an open-access article distributed under the terms of the Creative Commons Attribution License, which permits unrestricted use, distribution, and reproduction in any medium, provided the original author and source are credited. 
appearance. Particles determined as ACM had surfaces with compacted fibers and fibers exposed on the cross section. Particles determined as non-ACM consisted of heavy concrete or brick. Furthermore, there were some groups of CDW particles that had the same appearance, i.e., which appeared like the particles were derived from the same construction materials. For example, there were groups of gray corrugated sheets, brown brick, and concrete with white paint. Even though visual observation of fibers exposed on the cross section requires skill, grouping particles by visual appearance is very easy. For example, even if gray corrugated sheets and brown bricks are mixed, we can identify the particles at a glance and sort them manually in a short time. This suggests that the separation time of ACM from piles of CDW could be shortened by grouping by visual appearance (GVA) rather than DVL of individual particles.

In this study, the separation efficiency and the sorting time of CDW particles by GVA were determined. Using the results, in the case that the separation efficiency by GVA in this study was equivalent to that by DVL in the previous study, the reduction of sorting time by GVA was evaluated.

\section{Theory and Methods}

\section{Determination and sorting of asbestos-containing material}

In this study, a material is thought to contain asbestos if its asbestos content is $>0.1 \mathrm{w} \%$. ACM has to undergo special treatment prior to recycling or final disposal, as mentioned above. If ACM is present in CDW and the asbestos content in CDW is $>0.1 \mathrm{w} \%$, the whole CDW is regarded as ACM. However, because it is impossible to treat large amounts of CDW as ACM, it is necessary to convert CDW into nonACM by removing ACM. Furthermore, concentrating non-ACM promotes recycling of CDW.

Therefore, we considered ACM sorting in this study. Specifically, a CDW particle (called CDWP hereinafter) is selected and determined by visual observation as ACM or non-ACM. When the entire CDW has been sorted, a pile of CDWPs presumed to be ACM (ACMd: where " $\mathrm{d}$ " means "determined") and a pile of CDWPs presumed to be non-ACM (non-ACMd) will be made (Figure 1). Because ACMd and non-ACMd are determined by visual observation, human error is expected. From the point of view of separation efficiency, high-accuracy sorting of both ACMd and non-ACMd and a short sorting time are required.

\section{Separation efficiency, recovery rate, and asbestos content}

In regard to the determination of CDWP by visual observation, the determination as ACMd is considered "positive" (in the same way, the determination as non-ACMd is considered "negative") in this study. The total weights of truly ACM and non-ACM particles in CDWPs are called At and $\mathrm{Nt}$ ( $\mathrm{t}$ : truly A or $\mathrm{N}$ ), respectively. After sorting by visual observation, particles determined as ACMd and non-ACMd are called Ad and $\mathrm{Nd}$, respectively (d: determined). Because determination by visual observation naturally contains error, Ad consists of At and $\mathrm{Nt}$, i.e., contains both AtAd (determined as ACMd correctly) and NtAd (determined as ACMd regardless of truly non-ACM; false positive). In the same way, $\mathrm{Nd}$ contains both AtNd (determined as non-ACMd regardless of truly ACM; false negative) and $\mathrm{NtNd}$ (determined as nonACMd correctly). Therefore,

$$
\begin{aligned}
& \mathrm{At}=\mathrm{AtAd}+\mathrm{AtNd} \\
& \mathrm{Nt}=\mathrm{NtNd}+\mathrm{NtAd}
\end{aligned}
$$

After sorting:

Pile determined as positive Ad $=$ AtAd $+\mathrm{NtAd}$

Pile determined as negative $\mathrm{Nd}=\mathrm{AtNd}+\mathrm{NtNd}$

When the weight proportions of At and Nt to total CDWP's weight are defined as $\mathrm{WA}$ and $\mathrm{WN}$, respectively:

$$
\begin{aligned}
& \mathrm{WA}=\mathrm{At} /(\mathrm{At}+\mathrm{Nt}) \\
& \mathrm{WN}=\mathrm{Nt} /(\mathrm{At}+\mathrm{Nt})
\end{aligned}
$$

The rate of false negative determined from the positive sample group, i.e., false negative rate EA:

$$
\mathrm{EA}=\mathrm{AtNd} / \mathrm{At}
$$

The rate of false positive determined from the negative sample group, i.e., false positive rate $\mathrm{EN}$ :

$\mathrm{EN}=\mathrm{NtAd} / \mathrm{Nt}$

ACMa fresaned to be ACM



Figure 1: Concept of sorting CDW aiming at removal of ACM. 
Therefore,

$$
\begin{aligned}
& \mathrm{AtNd}=\mathrm{At} \cdot \mathrm{EA} \\
& \mathrm{AtAd}=\mathrm{At} \cdot(1-\mathrm{EA}) \\
& \mathrm{NtNd}=\mathrm{Nt} \cdot(1-\mathrm{EN}) \\
& \mathrm{NtAd}=\mathrm{Nt} \cdot \mathrm{EN}
\end{aligned}
$$

When we discuss the separation efficiency, not only the recovery rate of AtAd from At, but also the rate of AtNd contaminating Nd should be evaluated in order to avoid contaminating $\mathrm{Nd}$ with AtNd. Therefore, we consider Newton's separation efficiency, which can evaluate both rates.

Newton's separation efficiency $=$ AtAd $/$ At $+\mathrm{NtNd} / \mathrm{Nt}-1$

$$
\begin{aligned}
& =(\mathrm{At}-\mathrm{AtNd}) / \mathrm{At}+(\mathrm{Nt}-\mathrm{NtAd}) / \mathrm{Nt}-1 \\
& =1-\mathrm{NtAd} / \mathrm{Nt}-\mathrm{AtNd} / \mathrm{At} \\
& =1-\mathrm{EN}-\mathrm{EA}
\end{aligned}
$$

The recovery rates from At and $\mathrm{Nt}$ are defined as $\mathrm{RA}$ and $\mathrm{RN}$, respectively:

$$
\begin{aligned}
& \mathrm{RA}=\mathrm{AtAd} / \mathrm{At}=1-\mathrm{EA} \\
& \mathrm{RN}=\mathrm{NtNd} / \mathrm{Nt}=1-\mathrm{EN}
\end{aligned}
$$

When asbestos contents in At and Nt are defined as CA and CN, respectively, asbestos contents in $\mathrm{Ad}$ and $\mathrm{Nd}$ after sorting are called CAd and CNd:

$$
\begin{aligned}
& \mathrm{CAd}=(\mathrm{AtAd} \cdot \mathrm{CA}+\mathrm{NtAd} \cdot \mathrm{CN}) / \mathrm{Ad} \\
& \mathrm{CNd}=(\mathrm{AtNd} \cdot \mathrm{CA}+\mathrm{N} \mathrm{tNd} \cdot \mathrm{CN}) / \mathrm{Nd}
\end{aligned}
$$

When $\mathrm{CN}=0$, Equation (17) can be simplified:

$$
\mathrm{CNd}=\mathrm{AtNd} \cdot \mathrm{CA} / \mathrm{Nd}
$$

When the ratio of asbestos content $\mathrm{CNd}$ after sorting to original content CA is defined as content ratio RC:

$$
\mathrm{RC}=\mathrm{CNd} / \mathrm{CA}=\mathrm{AtNd} / \mathrm{Nd}=\mathrm{At} \cdot \mathrm{EA} / \mathrm{Nd}=\mathrm{EA} /(\mathrm{EA}+\mathrm{EN}(1 /
$$
$\mathrm{WA}-1)$ )

$$
\mathrm{CNd}=\mathrm{RC} \cdot \mathrm{CA}
$$

After we obtain RC, $\mathrm{CNd}$ can be calculated by assuming $\mathrm{CA}$ in Equation (20). The unit of RC is dimensionless. The units of CA and $\mathrm{CNd}$ are the same and optional, i.e., \% or dimensionless. Figure 1 shows the compositions of At and $\mathrm{Nt}$ before sorting, and $\mathrm{Ad}$ and $\mathrm{Nd}$ after sorting.

When the sorting times are defined as $\operatorname{tg}(\mathrm{h} / \mathrm{t})$ by GVA and $\mathrm{tl}(\mathrm{h} / \mathrm{t})$ by DVL:

$\operatorname{tg}=$ standard sorting time $(\mathrm{s} / \mathrm{p}) \cdot$ number of CDWPs $(\mathrm{p} / \mathrm{t}) / 602(21)$

$\mathrm{tl}=\left(\right.$ standard observation time $(\mathrm{s} / \mathrm{cm} 2) \cdot$ observation area $\left(\mathrm{cm}^{2} / \mathrm{p}\right)$ + shifting time $(\mathrm{s} / \mathrm{p})) \cdot$ number of CDWPs $(\mathrm{p} / \mathrm{t}) / 602$

(p: particle)

According to the authors' research [18], the standard observation time and the shifting time for DVL were $1.7 \mathrm{~s} / \mathrm{cm}^{2}$ and $1 \mathrm{~s} / \mathrm{p}$, respectively.

\section{Facilities and waste samples}

Waste samples were collected from an intermediate treatment facility in Japan where CDW was crushed and recyclable materials were recovered. The treatment flows at the demolition sites and the intermediate treatment facility are shown in Figure 2. Presorted ACMs were bagged $(\mathrm{X})$ at demolition sites. Other waste construction materials, i.e., non-ACMs, were manually presorted into recyclable

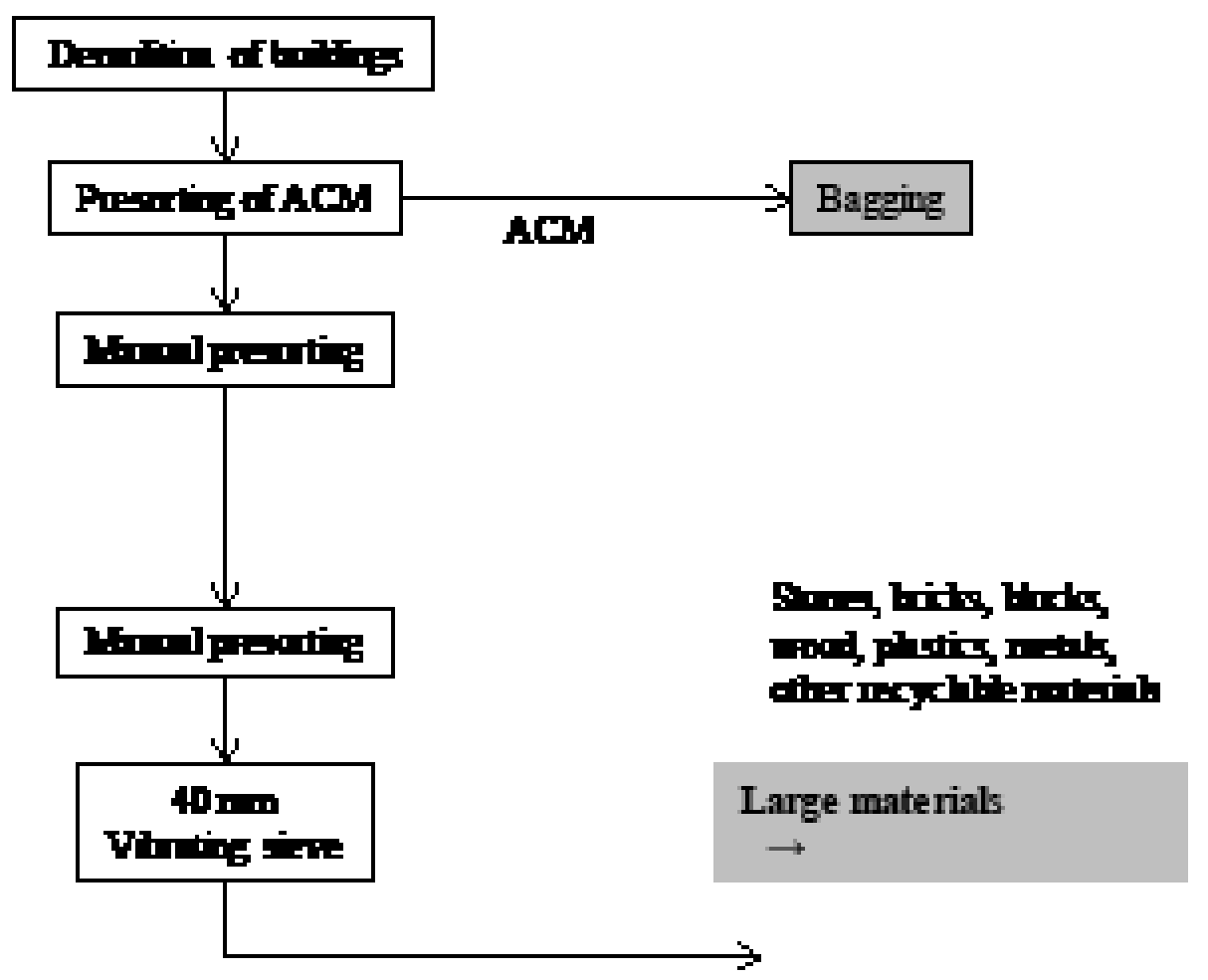

Figure 2: Treatment flows at demolition sites and intermediate treatment facility. Sampling points are also shown $(X$ and $Y)$ 
materials (e.g., stones and metals) and mixtures of other materials (hereinafter, "mixture"). The bagged ACMs (X), the recyclable materials, and the mixture were transported to the intermediate treatment facility. The bagged ACMs (X) were stored and brought to a landfill site. The mixture was manually presorted into recyclable materials and other residues. The residues were sieved through a 40 $\mathrm{mm}$ mesh vibrating sieve. Particles that remained on the sieve $(\mathrm{Y})$ were recycled or deposited at a landfill site, and fine particles that passed through the sieve were also deposited at a landfill site. $\mathrm{X}$ and $\mathrm{Y}$ were collected. Only sheetlike particles were collected from Y. However, there were few particles other than the sheetlike particles on the sieve. We thought that large particles were presorted into recyclable materials as stones, bricks, or blocks before sieving.

$\mathrm{X}$ and $\mathrm{Y}$ were mixed and 225 particles were sampled randomly. Dust on the particles was brushed off and the particles were washed with tap water. Then, the particles were numbered using an oil-based marking pen. A portion of the 225 particles were used in previous research $[18,19]$ and the other 127 CDW particles (hereinafter, referred to as "CDWP") were used in this study.

\section{Sample characteristics}

The dimensions (i.e., shortest, medium, and longest sides) and weight of CDWP were measured.

\section{Experimental methods}

Main points for determination of ACMd by visual appearance: ACMds were identified and sorted from CDWPs as follows: first, texture-like fibers compacted on the surface of CDWP were identified and then, fibers having the characteristics of asbestos on the cross section were identified, i.e., the asbestos fibers should be fascicular and non-uniform [18] (Figure 3). Therefore, an independent and uniform fiber (e.g., cotton or glass wool) is not asbestos (Figure 4).

Procedure for GVA: The results of GVA experiment conducted by several experimenters were required to calculate the separation efficiency of CDWP. Therefore, a GVA experiment was conducted with five experimenters. The experimenters were in their twenties and did not have any knowledge of the determination of ACM. The authors showed typical ACM characteristics and the above individual

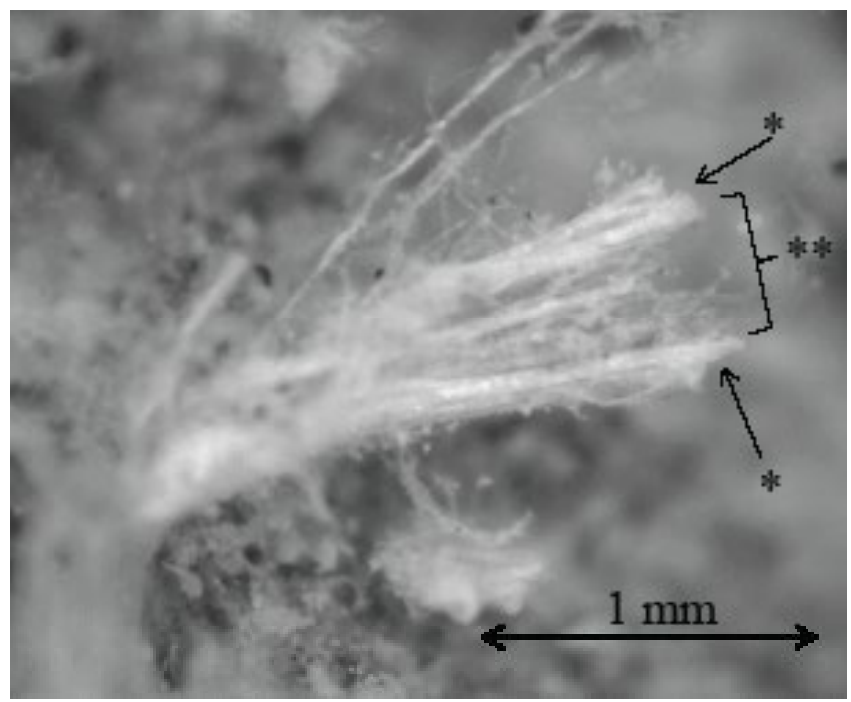

Figure 3: Typical asbestos fiber (chrysotile): fascicular* and non-uniform* ${ }^{\star *}$

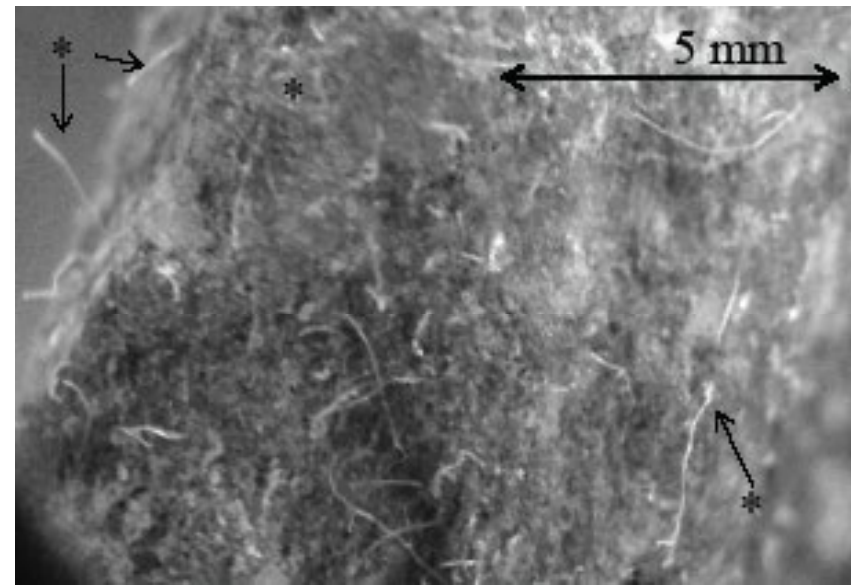

Figure 4: Typical non-asbestos fiber (glass or ceramic fiber): independent fibers*.

characteristics of asbestos fiber by using photographs for approximately 10 minutes. After that, the GVA experiment was performed as follows.

CDWPs in a tray were sorted into several groups according to visual appearance, such as surface texture, color, and fibers exposed on surface, as observed with the naked eye without a loupe. After that, the experimenters declared the group as ACMd, i.e., they sorted CDWPs into ACMd and other (non-ACM). For the group regarded as ACMd, the authors allowed the experimenters to confirm the typical characteristics of asbestos fiber. The total times for sorting and declaration were measured. After that, each sample number and its sorted group were recorded. All sorted CDWPs were re-mixed in the tray and another experimenter conducted the GVA experiment.

\section{Asbestos content}

Asbestos content in CDWP was measured after GVA experiment. The target minerals for measurement were chrysotile, amosite, crocidolite, tremolite, actinolite, and anthophyllite. Qualitative and quantitative analyses were conducted by transmission electron microscopy (EPA600/R-93/116) and the minimum determination limit was $0.1 \mathrm{w} \%$.

\section{Experimental Results}

\section{Sample characteristics}

The longest, medium, and shortest sides of a particle were $6.2 \mathrm{~cm}$, $4.0 \mathrm{~cm}$, and $1.2 \mathrm{~cm}$ on average, respectively, and the average weight was $66.6 \mathrm{~g}(\mathrm{n}=127)$.

\section{Asbestos content}

The number of samples in which asbestos content was $<0.1$ $\mathrm{w} \%$ (non-ACMs) was 89 . The number of samples in which asbestos content was $>0.1 \mathrm{w} \%$ (ACMs) was 38 , and the minimum, average, and maximum contents were $7.0 \mathrm{w} \%, 12 \mathrm{w} \%$, and $20 \mathrm{w} \%$, respectively. ACMs were composed of 27 particles from $X$ and 11 from $Y$. Therefore, ACMs consisted of not only typical ACMs presorted at demolition sites (X), but also ACMs contaminating mixture (Y) (Figure 2). Chrysotile was detected in all of the $38 \mathrm{ACM}$ samples, whereas crocidolite was detected in only one sample ( $4.0 \%$ of crocidolite, $10.0 \%$ of chrysotile) Amosite, tremolite, actinolite, and anthophyllite were not detected. By measuring asbestos content after GVA examination, each numbered 
Citation: Asakura H, Nakagawa K (2017) Grouping by Visual Appearance of Construction and Demolition Waste for Sorting Time Reduction with the Aim of Removing Asbestos-Containing Materials. Int J Waste Resour 7: 298. doi: 10.4172/2252-5211.1000298

Page 5 of 9

CDWP was finally identified as positive or negative. Photographs of particles determined as correctly positive (ACM) are shown in Figure 5. The names of construction material appearing on baggage $\mathrm{X}$ were Japanese product names of fiber-reinforced cement boards.
Photographs of particles determined as correctly negative (non-ACM) are shown in Figure 6. Concrete, concrete with tile, concrete with white paint, and brick were typical examples.

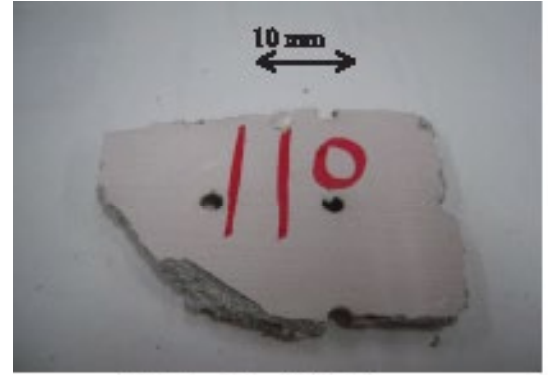

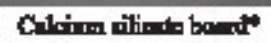

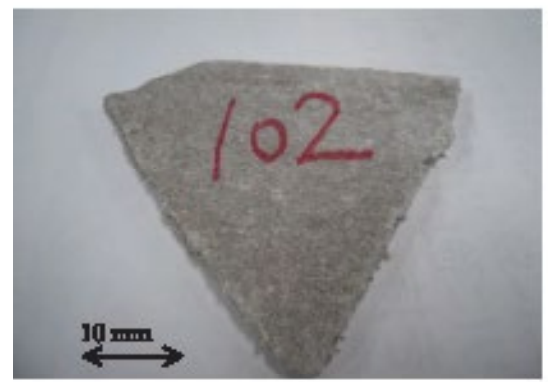

Note: *The name of construction material appears on the baggage
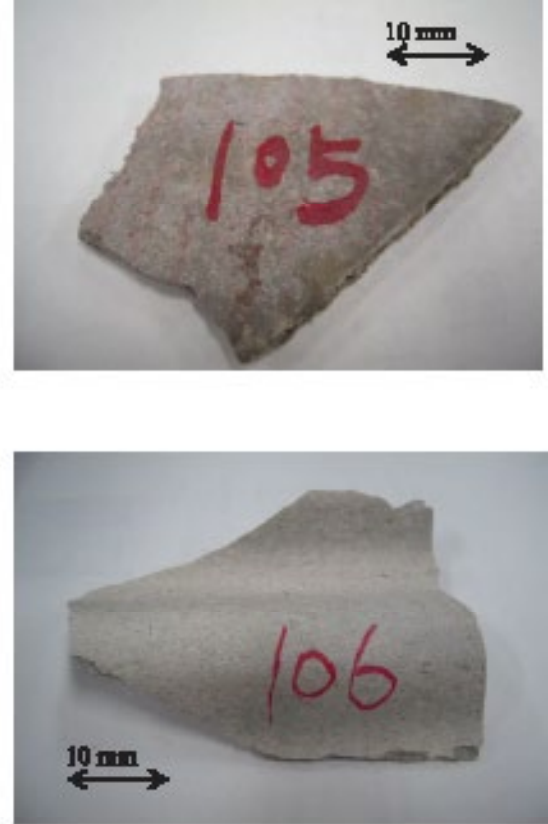

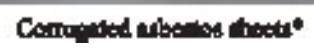

Figure 5: Particles determined as correctly positive (ACM).

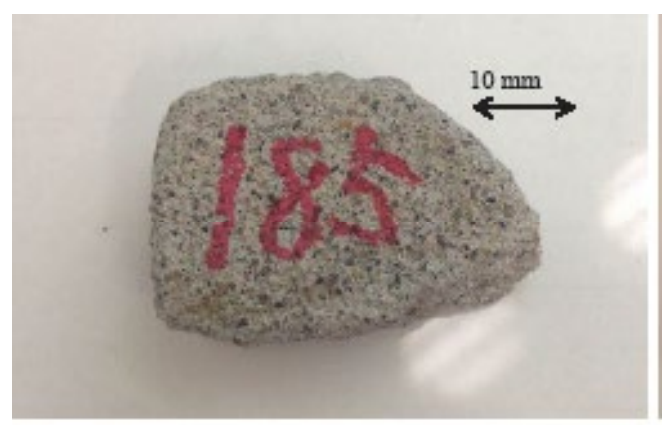

Conchit

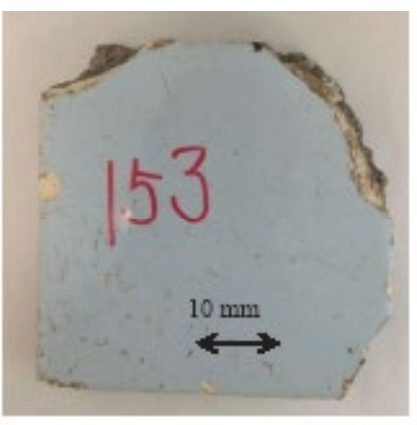

Concriar viili tile

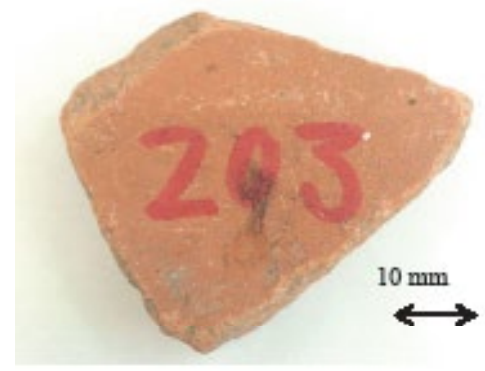

Firk

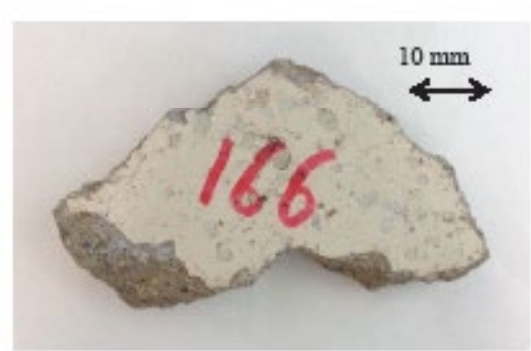

Concle oith ihiepict

Figure 6: Particles determined as correctly negative (non-ACM). 
Citation: Asakura H, Nakagawa K (2017) Grouping by Visual Appearance of Construction and Demolition Waste for Sorting Time Reduction with the Aim of Removing Asbestos-Containing Materials. Int J Waste Resour 7: 298. doi: 10.4172/2252-5211.1000298

Page 6 of 9

\section{False-positive/negative rates and separation efficiency by GVA}

False-positive rate EN, false-negative rate EA, and Newton's separation efficiency obtained by GVA are shown in Table 1. The averages of EN, EA, and Newton's separation efficiency were $0.02,0.02$, and 0.96, respectively. All ENs determined by the five experimenters were 0.022 and EAs ranged from 0 to 0.034 . The average sorting time of 127 particles was $175.6 \mathrm{~s}$, i.e., the standard sorting time was $1.4 \mathrm{~s} / \mathrm{p}$ (Table 1).

\section{Discussion}

\section{Characteristics of CDWP determined erroneously}

Two samples were determined as false positive (Figure 7). The samples had texture-like fibers compacted on the surface. Three samples were determined as false negative (Figure 8), and white paint was a common characteristic. From the point of view of ACM diffusion prevention, false negative in particular could be a problem. If ACMs

\begin{tabular}{|c|c|c|c|c|c|c|}
\hline \multicolumn{6}{|c|}{ Experimenter No. } & \multirow[t]{2}{*}{ Ave. } \\
\hline & 1 & 2 & 3 & 4 & 5 & \\
\hline EN & 0.022 & 0.022 & 0.022 & 0.022 & 0.022 & 0.02 \\
\hline EA & 0.011 & 0.011 & 0.034 & 0.022 & 0 & 0.02 \\
\hline NSE $^{*}$ & & & & & & 0.96 \\
\hline
\end{tabular}

Note: *Newton's separation efficiency

Table 1: False positive/negative rates and separation efficiency by GVA.
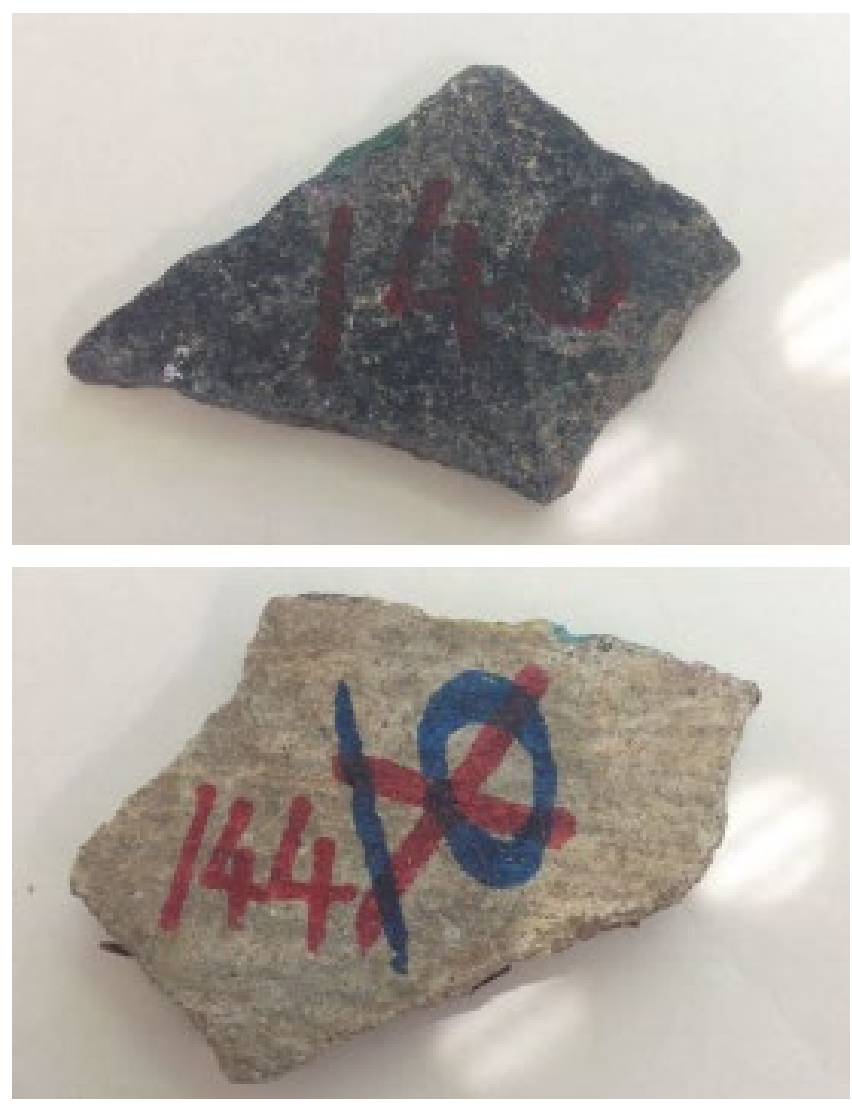

Figure 7: Particles determined as false positive, a: 5 / 5, b: 5 / 5 (error/ experimenter number). were painted or ornamented and there were similar CDWP in nonACMds, the ACMs could be erroneously determined as non-ACMd.

\section{Separation efficiency and sorting time}

With the average size obtained in section 4.1, the standard shape of CDWP was assumed as a disk consisting of an ellipse that had the longest $(6.2 \mathrm{~cm})$ and medium sides $(4.0 \mathrm{~cm})$, and the shortest side $(1.2$ $\mathrm{cm}$ ) as thickness, and its weight was $66.6 \mathrm{~g}$ (Figure 9). Therefore, ellipse area was $21.3 \mathrm{~cm}^{2}$, disk volume was $28.3 \mathrm{~cm}^{3}$, cross-sectional area was $20.6 \mathrm{~cm} 2$, and density was $2.4 \mathrm{~g} / \mathrm{cm}^{3}$. Hereinafter, the similar shape of disks was assumed even if the size of assumed CDWP changed (Figure 10).

We discuss separation efficiency and sorting time as follows. $1 \mathrm{t}$ of CDWPs having uniform, standard shape (longest side and weight are $6.2 \mathrm{~cm}$ and $66.6 \mathrm{~g} / \mathrm{p}$, respectively) is assumed, i.e., particle number is $15000 \mathrm{p} / \mathrm{t}$. Although the assumed CDWP size is optional, the size is assumed because the separation efficiency and sorting time are dependent on particle size.
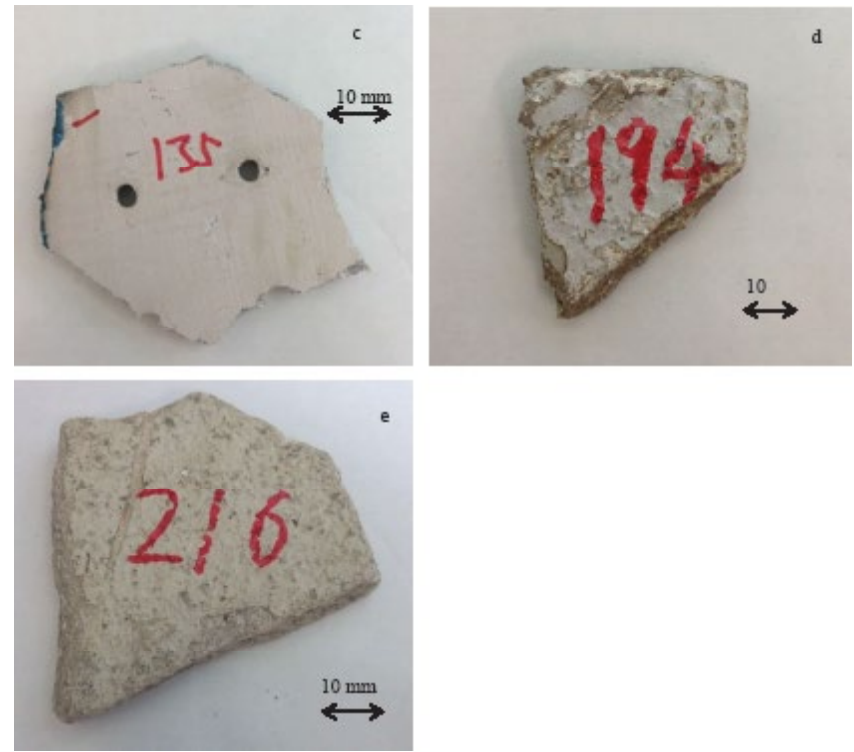

c: $15 \%$, d: $15 \%$, e: $10 \%$ (chrysotile).

Figure 8: Particles determined as false negative, c: 1 / 5, d: 4 / 5, e: 2 / 5 (error / experimenter number),

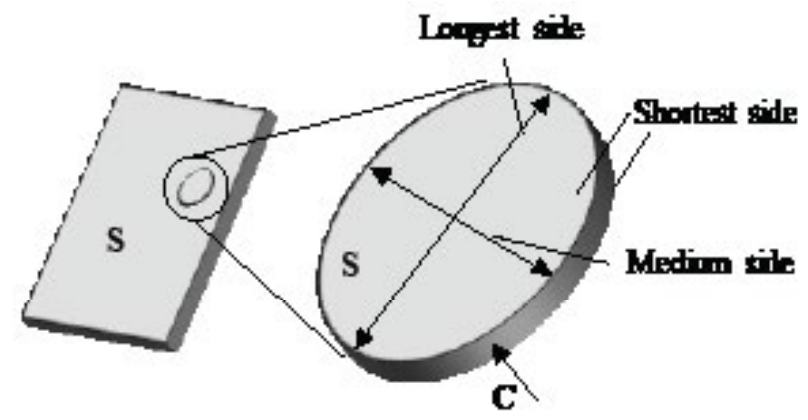

S: Shaped there

C. Cruss section

Figure 9: Assumed standard shape of $\mathrm{CDW}_{\mathrm{P}}$. 


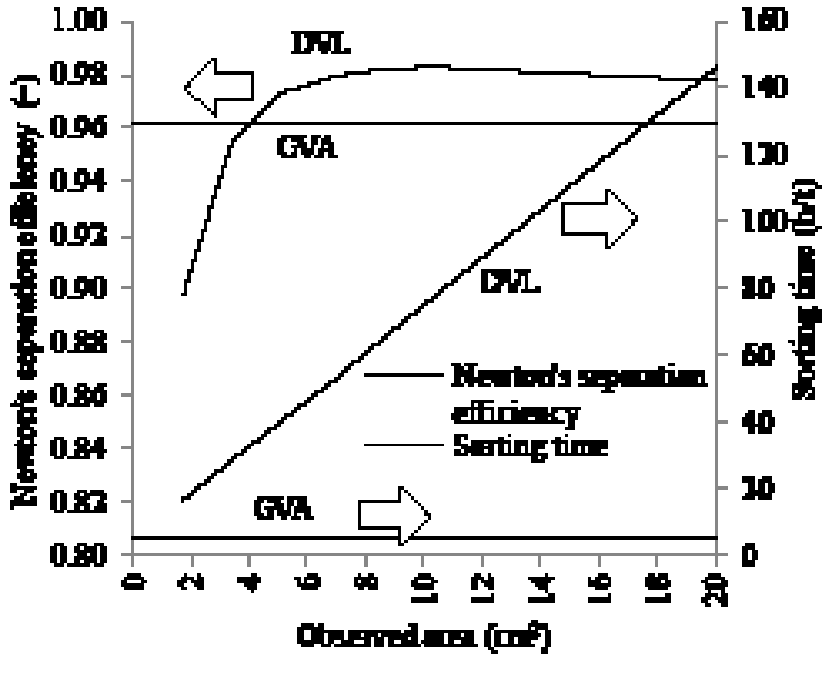

Figure 10: Separation efficiency and time.

In regard to GVA, Newton's separation efficiency and standard sorting time were 0.96 and $1.4 \mathrm{~s} / \mathrm{p}$ as mentioned above, i.e., the required sorting time was $1.4 \mathrm{~s} / \mathrm{p} \cdot 15000 \mathrm{p} / \mathrm{t}=5.8 \mathrm{~h} / \mathrm{t}$.

We discuss DVL as follows. Using the same samples as those in this study, the authors previously investigated the distribution of asbestos fiber bundles exposed on the cross section of CDWPs [19]. The cross section was divided into 12 parts, and the number of fiber bundles was counted in each section, i.e., the distribution of fiber bundles was obtained. From the results, false-negative and false-positive rates were calculated when observation was carried out on some sections of the cross sections. Fiber bundles were assumed to be exposed on $\mathrm{m}$ sections of the 12 sections, i.e., not exposed on 12 - $\mathrm{m}$ sections. In the observation on $\mathrm{k}$ sections of the 12 sections, the number of combinations was $12 \mathrm{Ck}$. The number of combinations in which fiber bundles were found on any $\mathrm{k}$ sections was $12 \mathrm{Ck}$ when $\mathrm{k}>(12-\mathrm{m})$, or $12 \mathrm{Ck}-(12-\mathrm{m}) \mathrm{Ck}$ when $\mathrm{k}$ $\leq(12-\mathrm{m})$. False-positive results were obtained when a particle had some fiber bundles (non-asbestos or asbestos fiber) regardless of nonACM and some sections having fiber bundles were observed, i.e., falsepositive rate EN was 1 when $\mathrm{k}>(12-\mathrm{m})$, or $1-(12-\mathrm{m}) \mathrm{Ck} / 12 \mathrm{Ck}$ when $\mathrm{k} \leq(12-\mathrm{m})$. False-negative results were obtained when some sections without fiber bundles were observed, i.e., false-negative rate EA was 0 when $\mathrm{k}>(12-\mathrm{m})$, or $(12-\mathrm{m}) \mathrm{Ck} / 12 \mathrm{Ck}$ when $\mathrm{k}<=(12-$ $\mathrm{m})$. Although only 12 and 6 of the 12 sections were discussed in the previous study [19], false-positive and false-negative rates of all section numbers (from 1 to 12 ) were calculated in this study.

Next, section number was converted into area for easy understanding of the observed size with a loupe. Assuming the standard shape of CDWP, area per unit section $\left(1.7 \mathrm{~cm}^{2}\right)$ was calculated, and section number (from 1 to 12 ) was converted into area (from 1.7 to $21 \mathrm{~cm}^{2}$ ). For example, the area of the 12 sections of the standard shape of CDWP was $20.6 \mathrm{~cm}^{2}$, and that of the 6 sections was half that value, i.e., 10.3 $\mathrm{cm}^{2}$. In addition, assuming that fiber bundles exposed on cross section were presented uniformly regardless of CDWP size, the relationship between observation area of CDWP larger than the standard shape and separation efficiency could be discussed.

In regard to DVL, Newton's separation efficiency was 0.90 for 1.7 $\mathrm{cm}^{2}$ observation, and more than 0.97 for $>5.1 \mathrm{~cm}^{2}$ observation. The reason was that the false-negative rate EA significantly decreased (asbestos fiber bundles were found) and observation area increased, even though the false-positive rate EN increased (sparse fiber bundles were found). Sorting time increased linearly with observation area (Equation (22)), i.e., $16 \mathrm{~h} / \mathrm{t}$ for $1.7 \mathrm{~cm}^{2}, 41 \mathrm{~h} / \mathrm{t}$ for $5.1 \mathrm{~cm}^{2}$, and $150 \mathrm{~h} / \mathrm{t}$ for $20.6 \mathrm{~cm}^{2}$ observation.

Separation efficiencies and sorting times by GVA and DVL are shown in Fig. 10. Newton's separation efficiency by GVA (0.96) was equivalent to that by DVL for $5.1 \mathrm{~cm}^{2}$ observation. Sorting time of $5.8 \mathrm{~h} / \mathrm{t}$ by GVA was $1 / 7$ of that by DVL ( $41 \mathrm{~h} / \mathrm{t})$. In this way, sorting time was shortened by GVA rather than DVL under the condition of equivalent separation efficiency.

\section{Asbestos contents after sorting and recovery rates}

Asbestos content ratios RCs after sorting are shown in Figure 11. According to Equation (19), RC is dependent on WA, i.e., the weight proportion of At. When WA increases, ACM contamination ratio in non-ACMd increases, i.e., $\mathrm{RC}$ also increases. In the following, WA is assumed to be 0.20 . RC for GVA is 0.0040 . RC for DVL changes depending on the observation area. When observation area increases, false-negative rate (contamination of ACM in non-ACMd) EA decreases, i.e., RC also decreases (Equation (19)). RCs are 0.024 for $1.7 \mathrm{~cm}^{2}$ observation and 0.0093 for $3.4 \mathrm{~cm}^{2}$ observation. Therefore, assuming $10 \%$ of $\mathrm{CA}$ (original asbestos content in $\mathrm{ACM}$ ), CNds (asbestos content in $\mathrm{Nd}$ after sorting) is $0.24 \%$ for $1.7 \mathrm{~cm}^{2}$ observation and $0.093 \%$ for $3.4 \mathrm{~cm}^{2}$ observation (Equation (20)), i.e., asbestos content in non-ACMd for $1.7 \mathrm{~cm}^{2}$ observation exceeds the standard value of $0.1 \%$. RC of 0.0040 for GVA is equivalent to RC of 0.0044 for DVL for $5.1 \mathrm{~cm}^{2}$ observation. Assuming $10 \%$ of CA, CNd is $0.04 \%$, i.e., below the standard value.

The recovery rate RA of ACM is 0.98 for GVA (Figure 12). In regard to DVL, when observation area increases, false-negative rate EA decreases, i.e., RA also increases (Equation (14)). RA of 0.98 for GVA is equivalent to RA for DVL for $5.1 \mathrm{~cm}^{2}$ observation.

\section{Relationship between particle size and sorting time}

The relationship between particle size and sorting time is shown in Figure 13. Particle weight was calculated as follows. At first, a disk-

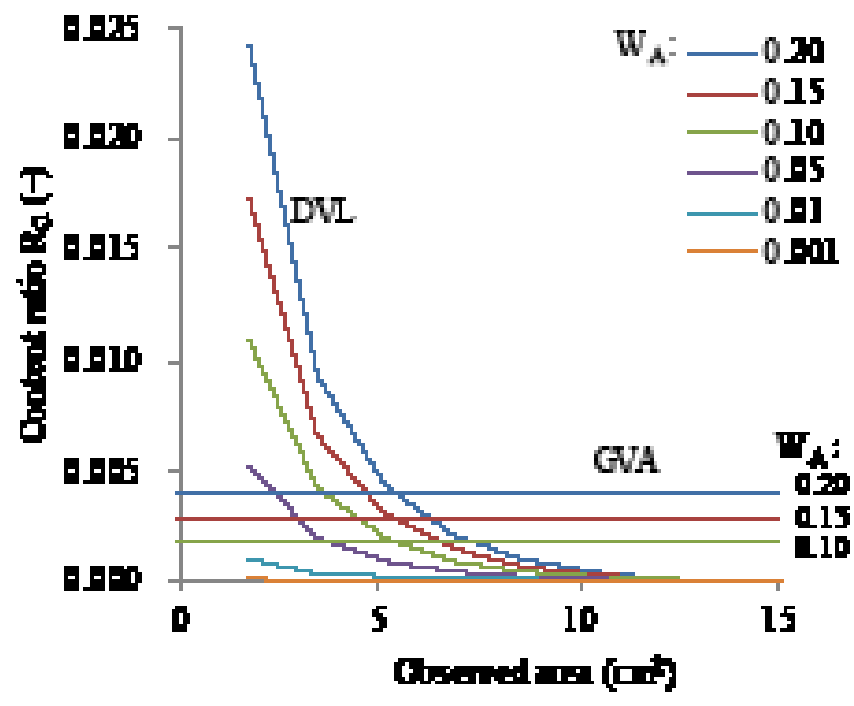

Figure 11: Asbestos content ratio $R_{c}$ after sorting. 


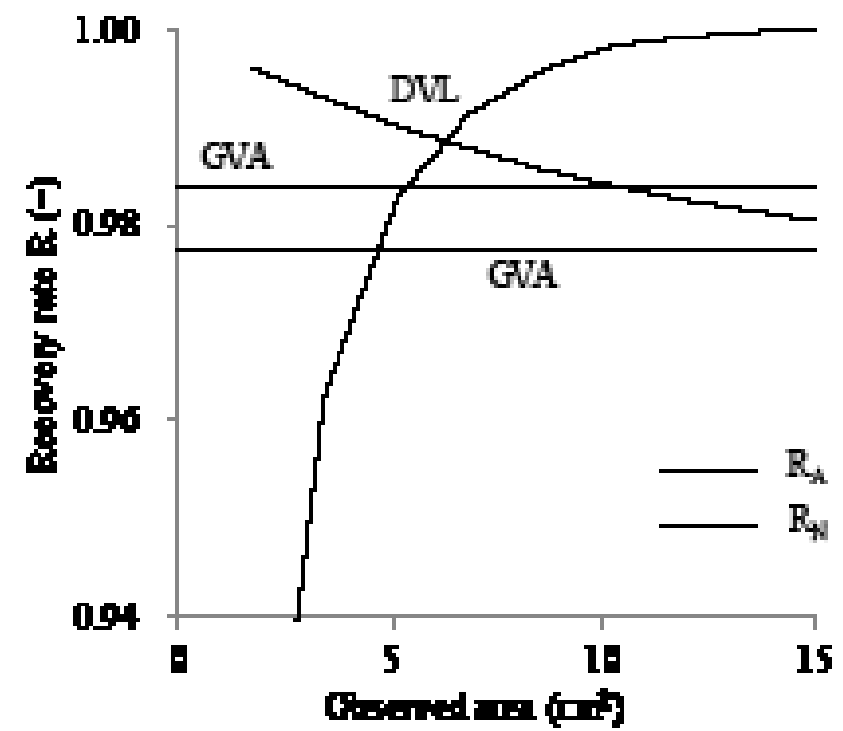

Figure 12: Recovery rate.

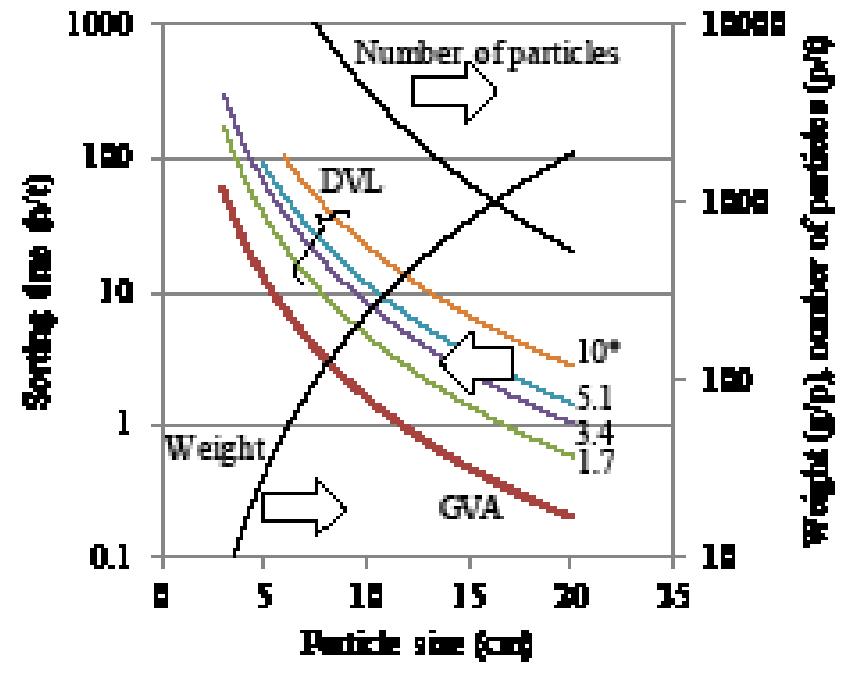

Note: *Observation area using loupe $\left(\mathrm{cm}^{2}\right)$

Figure 13: Relationship between particle size and sorting time

shaped particle similar to the standard shape of CDWP was assumed, and the volume of the assumed particle was calculated by setting any particle size (longest side). Next, the weight of the assumed CDWP was obtained from the density $\left(2.4 \mathrm{~g} / \mathrm{cm}^{3}\right)$. The CDWP weights for $2 \mathrm{~cm}, 5$ $\mathrm{cm}, 10 \mathrm{~cm}, 20 \mathrm{~cm}$, and $30 \mathrm{~cm}$ particles were $2.2,34,270,2170$, and 7300 $\mathrm{g} / \mathrm{p}$, respectively. The number of particles per ton was $4.6 \times 10^{5}, 2.9 \times 10^{4}$, 3700,460 , and 140 , respectively.

Sorting time was calculated from particle size (Equations (21) and (22)). Regardless of particle size, the sorting time by GVA was approximately $1 / 7$ of that by DVL for $5.1 \mathrm{~cm}^{2}$ observation. In regard to CDWP with $20 \mathrm{~cm}$ particle size, sorting time was $0.2 \mathrm{~h} / \mathrm{t}$ by GVA, and $0.58\left(1.7 \mathrm{~cm}^{2}\right.$ observation) to $2.7\left(10 \mathrm{~cm}^{2}\right.$ observation $) \mathrm{h} / \mathrm{t}$ by DVL. In regard to CDWP with $10 \mathrm{~cm}$ particle size, sorting time was $1.6 \mathrm{~h} / \mathrm{t}$ by GVA, and $4.6\left(1.7 \mathrm{~cm}^{2}\right.$ observation $)$ to $22\left(10 \mathrm{~cm}^{2}\right.$ observation $) \mathrm{h} / \mathrm{t}$ by
DVL. In order to reduce sorting time per worker to less than $1 \mathrm{~h} / \mathrm{t}$, only CDWPs having particle size exceeding $12 \mathrm{~cm}$ for GVA, or $20 \mathrm{~cm}$ for DVL for $5.1 \mathrm{~cm}^{2}$ observation should be subjected to sorting. Therefore, considering sorting time, small CDWP could not be sorted even by visual observation (Figure 13).

\section{Suggestion for effective separation}

In this study, the applicability of ACM separation from CDW by GVA was shown. However, the authors do not recommend that CDW be regarded as non-ACMd for subsequent treatment and recycling without performing any standard tests. Piles of non-ACMds should be subjected to some kind of standard test for asbestos content. The results of this study should be limited to the discussion of GVA's superior speed and efficiency in CDW separation. Based on the results of this study, aiming to avoid diffusion of asbestos-containing waste, the authors suggest that grouping by visual appearance as the primary sorting step is effective to reduce sorting time when CDW from disaster waste or unknown origin has to be sorted.

\section{Conclusion}

Separation efficiency and sorting time of CDW particles by GVA were determined. In the case that separation efficiency by GVA in this study was equivalent to that by DVL in the previous study, the reduction of sorting time by GVA was evaluated. The main findings were as follows:

The averages of false-positive rate EN, false-negative rate EA, Newton's separation efficiency, and standard sorting time for GVA were $0.02,0.02,0.96$, and $1.4 \mathrm{~s} / \mathrm{p}$. CDWP erroneously determined as ACMd by GVA had texture-like fibers compacted on the surface. On the other hand, the reason why non-ACMd was erroneously determined could be that ACMs were painted or ornamented and there were similar CDWP in non-ACMds. Newton's separation efficiency (0.96), asbestos content ratio RC, and recovery rate RA for GVA were equivalent to those for DVL for $5.1 \mathrm{~cm}^{2}$ observation. Sorting time by GVA was $1 / 7$ of that by DVL. Sorting time could be shortened by GVA compared with that by DVL under the condition of equivalent separation efficiency. In order to reduce sorting time per worker to less than $1 \mathrm{~h} / \mathrm{t}$, only CDWPs having particle size exceeding $12 \mathrm{~cm}$ for GVA, or $20 \mathrm{~cm}$ for DVL for 5.1 $\mathrm{cm}^{2}$ observation should be subjected to sorting.

In this study, the applicability of ACM separation from CDW by GVA was shown. Aiming to avoid diffusion of asbestos-containing waste, the authors suggest that grouping by visual appearance as the primary sorting step is effective to reduce sorting time when CDW from disaster waste or unknown origin has to be sorted.

\section{Acknowledgements}

The authors thank the Ministry of Environment for a "Grant-in-Aid for Scientific Research on Waste Treatment" under the project "Establishment of screening methods of asbestos-containing construction materials, and safety assessment of recycled crushed stone (FY 2011 to 2013). And this work was supported by JSPS KAKENHI (Grants-in-Aid for Scientific Research C) Grant Number JP17K00600.

\section{References}

1. EC (2001) Commission Decision of 16 January 2001 amending Decision 2000/532/EC as regards the list of wastes (2001/118/EC)

2. Ministry of Health, Labour and Welfare in Japan (1972) Order for enforcement of industrial safety and health act, Article 6. Tokyo, Japan (in Japanese).

3. US EPA (1990) Regulated asbestos-containing materials guidance. EPA340/1-90-018.

4. Saitama Japan Industrial Waste Association (2011) Safety management manual for aggregate recycling. Saitama, Japan (in Japanese). 
Citation: Asakura H, Nakagawa K (2017) Grouping by Visual Appearance of Construction and Demolition Waste for Sorting Time Reduction with the Aim of Removing Asbestos-Containing Materials. Int J Waste Resour 7: 298. doi: 10.4172/2252-5211.1000298

5. Bishop K, Ring SJ, Zoltai T, Manos CG, Ahrens VD, et al. (1985) Identification of asbestos and glass fibers in municipal sewage sludges. Bull Environ Contam Toxicol 34: 301-308.

6. Tadas P, Dainius M, Edvinas K, Linas K, Maksim K, et al. (2011) Comparative characterization of particle emissions from asbestos and non-asbestos cement roof slates. Building and Environment 46: 2295-2302.

7. Plescia P, Gizzi D, Benedetti S, Camilucci L, Fanizza C, et al. (2003) Mechanochemical treatment to recycling asbestos-containing waste. Waste Management 23: 209-218.

8. Min S, Maken S, Park J, Gaur A, Hyun J (2008) Melting treatment of waste asbestos using mixture of hydrogen and oxygen produced from water electrolysis. Korean J Chem Eng 25: 323-328.

9. Zaremba T, Peszko M (2008) Investigation of the thermal modification of asbestos wastes for potential use in ceramic formulation. J Therm Anal Calorim 92: 873-877.

10. Gualtieri AF, Giacobbe C, Sardisco L, Saraceno M, Gualtieri ML, et al. (2011) Recycling of the product of thermal inertization of cement-asbestos for various industrial applications. Waste Management 31: 91-100.

11. Osada M, Takamiya K, Manako K, Noguchi M, Sakai S (2013) Demonstration study of high temperature melting for asbestos-containing waste (ACW). J Mater Cycles Waste Manag 15: 25-36.

12. Radvanec M, Tuček L', Derco J, Čechovská K, Németh Z (2013) Change of carcinogenic chrysotile fibers in the asbestos cement (eternit) to harmless waste by artificial carbonatization: Petrological and technological results. J Hazard Mater.

13. Ruud CO, Barrett CS, Russell PA, Clark RL (1969) Selected area electron diffraction and energy dispersive $\mathrm{X}$-ray analysis for the identification of asbestos fibres, a comparison. Micron 7: 115-132.

14. Pooley FD (1975) The identification of asbestos dust with an electron microscope microprobe analyser. Ann Occup Hyg 18: 181-186.

15. Taylor DG, Baron PA, Shulman SA, Carter JW (1984) Identification and counting of asbestos fibers. Am Ind Hyg Assoc J 45: 84-88.

16. Bard D, Yarwood J, Tylee B (1997) Asbestos fibre identification by Raman microspectroscopy. J Raman Spectrosc 28: 803-809.

17. Bassani C, Cavalli RM, Cavalcante F, Cuomo V, Palombo A, et al. (2007) Deterioration status of asbestos-cement roofing sheets assessed by analyzing hyperspectral data. Remote Sensing of Environment 109: 361-378.

18. Asakura H, Kawasaki M, Suzuki K, Nakagawa K, Watanabe Y (2014) Determination and sorting of asbestos-containing material by visual observation. American Journal of Environmental Protection 3: 275-282.

19. Asakura H, Kawasaki M, Suzuki K, Nakagawa K, Watanabe Y (2014) Difference in density of fiber bundles exposed on surface of asbestos-containing materials with the aim to reduce time necessary for visual observation. International Journal of Environmental and Resource 3: 46-53. 